\title{
Studies on the fate of $\mathrm{D}$-aspartic acid in pineal and pituitary glands of rats after intravenous administration
}

\author{
By Takeshi Fukushima, Mika ShiraO, Hideaki IchiHara, Tomofumi Santa, Hiroshi Homma, \\ and Kazuhiro $\mathrm{IMAI}^{\dagger}$ \\ Graduate School of Pharmaceutical Sciences, The University of Tokyo, 7-3-1 Hongo, Bunkyo-ku, Tokyo 113-0033 \\ (Communicated by Masanori OTsuKA, M. J. A., Jan. 12, 1998)
}

\begin{abstract}
The present study was intended to confirm the idea that D-aspartic acid (Asp) in pineal and pituitary glands could originate from diet as suggested by our previous report [Imai et al. (1997) Proc. Japan Acad. 73B, 48-52]. When the rats were kept under fasting for 2 or 3 days, the levels of D-Asp, but not L-Asp in these glands were significantly lower as compared with those in the rats with diet. The fact suggests that D-Asp in these glands is derived from the exogenous origin. Next, we determined the D- or L-Asp concentration in these glands (at $30 \mathrm{~min}, 3 \mathrm{hr}$ and $24 \mathrm{hr}$ ) and plasma ( $2 \mathrm{~min}-5 \mathrm{hr}$ ) following intravenous administration of D- or LAsp $(50 \mathrm{mg} / \mathrm{kg}$ ) into rats. D-Asp concentrations remarkably increased after administration of D-Asp, and the levels were almost constant from $30 \mathrm{~min}$ to $24 \mathrm{hr}$ after administration, indicating that D-Asp was accumulated and deposited in these glands. In contrast, no accumulation of L-Asp was observed in case of L-Asp or D-Asp administration. In plasma, either D- or L-Asp concentration decreased to the normal level at $3 \mathrm{hr}$ after the administration of the respective enantiomers, although different half-lives for D- and L-Asp in plasma were obtained; $7.84 \mathrm{~min}$ for D- and $3.75 \mathrm{~min}$ for L-Asp. These results indicate that D-Asp incorporated from extracellular fluids into pineal and pituitary glands is difficult to be metabolized and eliminated, and strongly support the idea that the D-Asp in these glands in vivo is derived from the exogenous source, such as dietary DAsp.
\end{abstract}

Key words : D-aspartic acid; intravenous administration; fasting; rat; pineal gland; pituitary gland.

Introduction. Although D-amino acid has long been recognized as an unnatural type of amino acid, ${ }^{1)}$ the recent advances in chromatographic techniques of enantiomeric separation and sensitive detection have allowed the occurrence of $\mathrm{D}$-amino acids in tissues or biological fluids of mammals ${ }^{2)}$ to be found and accurately measured. Among them, D-aspartic acid (Asp) $)^{3)-8)}$ and D-serine $\left(\right.$ Ser) ${ }^{7)-10)}$ have been well studied because of their selective distribution in the mammalian tissues. D-Ser has a potent binding activity to the NMDA strychinineinsensitive glycine binding site in brain and is now considered to be an intrinsic ligand for the NMDA receptor. ${ }^{8), 9)}$ Recently, the biosynthesis of D-Ser in rat brain through racemization of L-Ser was also suggested. ${ }^{10)}$

As for D-Asp, there are some reports on the occurrence in endocrine organs ${ }^{5)}$ such as pituitary gland, testis and adrenal gland, and its distinctive pattern of emergence in the life cycle of each respective tissues of

t) Correspondence to: K. Imai. the central nervous system and periphery. ${ }^{8)}$ We found an extremely high concentration of D-Asp in rat pineal gland, ${ }^{11), 12)}$ and the change of concentration with age was also observed. Hence, D-Asp is considered to be closely related to the genesis or maturation of the mammalian tissues or organs. Recently, Schell et al. also reported the occurrence of D-Asp in rat pineal gland, ${ }^{13)}$ and considered that D-Asp functions as a novel neurotransmitter, or a modulator of steroid biosynthesis.

However, the origin of D-Asp in several tissues mentioned above is still ambiguous. D'Aniello et al. reported that radioactivity was detected in the whole brain of mice fed with the diet containing ${ }^{3} \mathrm{H}-\mathrm{D}$-Asp. ${ }^{4)} \mathrm{We}$ administered radiolabelled D-Asp $\left({ }^{14} \mathrm{C}\right.$-D-Asp) to rats intravenously, and investigated the distribution of radioactivity by whole body autoradiography (ARG) to identify the tissues concerned. ${ }^{14)}$ It was found that a high level of radioactivity was incorporated and accumulated in the rat pineal gland, followed by pituitary gland and other peripheral tissues, adrenal gland and testis, ${ }^{14)}$ where the 
amounts of D-Asp were high in vivo. ${ }^{5), 11), 12)}$ Thus, it was considered that D-Asp present in vivo in those tissues is originated from the daily diet containing D-Asp (a rat chow, CE-2, contains $50 \mathrm{nmol} \mathrm{D}-\mathrm{Asp} / \mathrm{g}$ ). ${ }^{15}$ )

To further clarify this issue, since there is a possibility that the radioactivity detected might include that of the metabolite, in the present work, we administer cold D- or L-Asp instead of radiolabelled Asp to male rats to investigate the fate of the intact D- or L-Asp in pineal and pituitary glands adopting high-performance liquid chromatographic determination and identification. We also investigate the effect of fasting on the concentration of DAsp in these tissues in vivo to search for its origin. Discussion will be made on the accumulation and elimination of D- or L-Asp in these glands.

Materials and method. Chemicals. 4-Fluoro-7nitro-2,1,3-benzoxadiazole (NBD-F) was purchased from Tokyo Kasei, Co., Ltd. (Tokyo, Japan). Sumichiral OA2500 (S) $(250 \times 4.6 \mathrm{~mm}$ i.d., $5 \mu \mathrm{m}$, Lot. No. OA251877) and (R) (Lot. No. OA251773) were kindly supplied by Sumika Chemical Analysis Service, Ltd. (Osaka, Japan). A TSK guardgel ODS-80Ts $(1.5 \times 3.2 \mathrm{~mm}$ i.d., $5 \mu \mathrm{m})$ was from Tosoh, Co., Ltd. (Tokyo, Japan). Methanol (MeOH) and acetonitrile $\left(\mathrm{CH}_{3} \mathrm{CN}\right)$ were of HPLC grade, and purchased from Kanto Pure Chemicals. Co., Ltd. (Tokyo, Japan). D-Asp was purchased from Sigma, Co., Ltd. (St. Louis, MO, U.S.A.), and L-Asp was from Wako Pure Chemicals. (Tokyo, Japan). Citrate monohydrate used was an analytical grade from Kanto Pure Chemicals.

Animal experiment. Male Sprague-Dawley rats (7week-old, Nihon Charles River, Kanagawa, Japan) were housed in an environmentally controlled room with a $12 \mathrm{hr}$ light/dark cycle, and provided with tap water and a commercial chow diet (type CE-2, Clea Japan, Tokyo, Japan) ad libitum. D- or L-Asp dissolved in saline (Otsuka Pharmaceuticals, Co., Ltd., Tokushima, Japan) was administered via a tail vein $(50 \mathrm{mg} / 10 \mathrm{ml} / \mathrm{kg})$ using a disposable syringe (Terumo, Tokyo, Japan) with a 25 gauge needle. The saline instead of D- or L-Asp solution was administered $(10 \mathrm{ml} / \mathrm{kg})$ as a control experiment.

Analysis in pineal and pituitary glands of rats under non-fasting and fasting. Twelve rats were divided into 3 groups, which were non-fasting, fasting for 2 days and 3 days. The rats were anesthetized with diethyl ether, and the blood was drawn from the abdominal aorta, and then, pineal and pituitary glands were removed quickly, and frozen at $-20^{\circ} \mathrm{C}$ until analysis. The glands dissected were homogenized in $\mathrm{MeOH}$ with cooling on ice, and then, the homogenate obtained was centrifuged at 3,000 rpm for 5 $\min$. One hundred $\mu \mathrm{L}$ for pituitary gland and $200 \mu \mathrm{L}$ for pineal gland of the resultant supernatant was evaporated to dryness using a centrifugal evaporator, SPE-200 (Shimad$z u$, Osaka, Japan). The residue was dissolved in $20 \mu \mathrm{L}$ of $0.2 \mathrm{M}$ borate buffer (pH 8.0) containing $4 \mathrm{mM}$ EDTA.2Na, and $30 \mu \mathrm{L}$ of $50 \mathrm{mM}$ NBD-F in $\mathrm{CH}_{3} \mathrm{CN}$ was added. The reaction mixture was heated at $60^{\circ} \mathrm{C}$ for $5 \mathrm{~min}$, and $950 \mu \mathrm{L}$ of $20 \mathrm{mM}$ citrate in $\mathrm{MeOH}$ was added. Five $\mu \mathrm{L}$ of the solution was injected into the HPLC with Sumichiral OA2500 (S) or (R).

Analysis in pineal and pituitary glands of rats after administration of D- or L-Asp. In this experiment, all rats used were under fasting for 2 days, and 3 rats were sacrificed at time points of $30 \mathrm{~min}, 3 \mathrm{hr}$ and $24 \mathrm{hr}$ after administration. The rats were anesthetized with diethyl ether, and the blood was drawn from the abdominal aorta and then, pineal gland and pituitary gland were removed quickly, and frozen at $-20^{\circ} \mathrm{C}$ until analysis. The glands dissected were treated in a similar manner described above.

Analysis in plasma of rats after administration of $D$ - or L-Asp. At each time point of 2, 15, 30, $60 \mathrm{~min}, 2,3$ and $5 \mathrm{hr}$ for D-Asp, and of 2, 15, $30 \mathrm{~min}$ and $3 \mathrm{hr}$ for L-Asp administration, $0.5 \mathrm{ml}$ of blood was drawn from a jagular vein using a heparinized syringe with a 25 gauge needle $(\mathrm{N}=3)$. Subsequently, the plasma sample was obtained by centrifugation of the blood at 4,000 rpm for $15 \mathrm{~min}$. Fifty $\mu \mathrm{L}$ of plasma was added to $450 \mu \mathrm{L}$ of $\mathrm{MeOH}$, and mixed vigorously. One hundred $\mu \mathrm{L}$ of the resultant supernatant was evaporated to dryness using SPE-200, and the residue was treated in a similar manner described above.

HPLC apparatus. HPLC used in this study consists of an intelligent pump, BIP-1 (Jasco, Tokyo, Japan), a fluorometric detector, L-7480 (Hitachi, Tokyo, Japan) and a chromatointegrator, D-7500 (Hitachi, Tokyo, Japan). A TSK guardgel ODS-80Ts was connected to the top of the chiral column [Sumichiral OA-2500 (S) or (R)]. The mobile phase employed was $8.0 \mathrm{mM}$ citric acid in $\mathrm{MeOH}$ for the analysis of pineal gland and $6.5 \mathrm{mM}$ for pituitary gland and plasma. The flow rate was at $1.0 \mathrm{ml} / \mathrm{min}$, and the column temperature was ambient. The fluorometric detection was made at $530 \mathrm{~nm}$ with $470 \mathrm{~nm}$ as an excitation wavelength.

Calibration curve. Standard solutions of D- and L-Asp were prepared at the concentration of $200,100,50,25$, $12.5,6.25,3.12,1.56 \mu \mathrm{M}$ in $0.2 \mathrm{M}$ borate buffer ( $\mathrm{pH} 8.0$ ) containing $4 \mathrm{mM}$ EDTA.2Na. Twenty $\mu \mathrm{L}$ of the solution was treated in the same way as for the sample from the biological materials.

Statistical analysis. All data cited in the text were expressed as mean $\pm \mathrm{SD}$, and significant differences between the groups were determined by Duncan's multiple 
range test. Half-life of D- or L-Asp in plasma was calculated by a computer software, MULTI using damping Gauss-Newton method (1-compartment model).

Results. As shown in Table I, the D-Asp concentrations in pineal and pituitary glands decreased significantly under fasting for 2 or 3 days, as did the body weights. In contrast, L-Asp concentration did not change even at 2 or 3 days. These data support the idea that D-Asp in pineal and pituitary glands occurring in vivo may be derived from external source, since the daily diet contains D-Asp, ${ }^{15}$ whereas L-Asp may be from the product of in vivo biosynthesis as well as from diet.

Considering the results, the next experiment of the intravenous administration of D-Asp or L-Asp into the rat fasted for 2 days was performed in order to augment the incorporation. When $5 \mathrm{mg}$ of D-Asp / $\mathrm{kg}$ was administered, no difference from the basal level of $\mathrm{D}$-Asp concentration in these glands were detected. Thus, a dose of $50 \mathrm{mg}$ of Dor L-Asp / $\mathrm{kg}$ was administered intravenously via the tail vein. Fig. 1 shows the typical HPLC [Sumichiral OA$2500(\mathrm{~S})]$ chromatograms of the methanol extracts obtained from pineal gland at $30 \mathrm{~min}$ after the administration of saline (control) (a), D-Asp (b) and L-Asp (c), respectively. The increased large peak corresponding to D-Asp derivative was clearly observed in the extract from pineal gland of rat to which D-Asp was administered (b). The peak areas for L-Asp in the chromatograms obtained from every rats to which D-Asp or L-Asp was administered were similar to those obtained from the control rats. The peak was identified as NBD-D-Asp or -L-Asp because of the same retention times as the standard NBD-D-Asp or -L-Asp and also the same retention times (48 min and 44 min, respectively) as the standard by using the chiral column having the opposite chiral center [Sumichiral OA2500(R)].

The amounts of D- and L-Asp obtained in these glands are summarized in Fig.2. As shown in Fig.2 (upper part), $D$-Asp concentrations in pineal gland increased extraordinarily from the control level $(p<0.01)$, and continued to be high at the time points examined ( 3 and $24 \mathrm{hr}$ ). The same trend was also true for D-Asp concentration in pituitary gland although the level slightly decreased at $24 \mathrm{hr}$ (Fig.2, lower part). The data indicate that the $\mathrm{D}$-Asp incorporated was stable for a long period of time in these glands. In the case of L-Asp administration, the accumulation of L-Asp was not observed at any time points examined in the glands. The data also show that the administration of $\mathrm{D}$ - or L-Asp did not affect so much the concentration of L- or DAsp, respectively, in both the glands. Although D'Aniello et al. mentioned the increase and long deposit of D-Asp in pituitary gland and testis of rats with an intraperitoneal administration of D-Asp (0.5-4.0 $\mu \mathrm{mol} / \mathrm{g}$ body weight), no quantitative data on time-course were reported in the text. ${ }^{6)}$

The time-course studies on D- or L-Asp in plasma revealed that the elimination rate of $\mathrm{D}$-Asp in plasma was apparently slower than that of L-Asp, whereas the levels of both Asp at 2 min were almost identical (Fig. 3). The halflife of D-Asp in plasma was $7.84 \mathrm{~min}$ and its concentration continued to decrease gradually at least until $24 \mathrm{hr}$ to reach the basal level (almost null). In contrast, the half-life of LAsp was $3.75 \mathrm{~min}$ and its concentration reached at a steady state from $30 \mathrm{~min}$, which was within the normal range of its concentration of $6.90 \pm 2.75 \mathrm{nmol} / \mathrm{ml}$ plasma obtained by the control experiment with saline administration. Although D-Asp concentrations in plasma after $3 \mathrm{hr}$ were already very low as compared to those at $30 \mathrm{~min}$, those in pineal and pituitary glands at 3 and $24 \mathrm{hr}$ were quite similar to those at $30 \mathrm{~min}$ (Fig. 2). The data suggest that D-Asp was incorporated from plasma into these glands and stayed for a long period of time in them.

Discussion. In the previous study, we administered ${ }^{14} \mathrm{C}-\mathrm{D}$-Asp into rats intravenously and investigated its distribution into the tissues by whole body ARG. The results showed that radioactivity was accumulated and deposited in peripheral tissues such as adrenal and testis and the distinct parts of brain, pineal and pituitary glands. ${ }^{14)}$ Considering the data, it was assumed that a part

Table I. D- and L-Asp concentration in pineal and pituitary glands as well as body weight of rats under fasting for 2 and 3 days

\begin{tabular}{|c|c|c|c|c|c|}
\hline \multirow[b]{3}{*}{ Control } & \multicolumn{2}{|c|}{ Pineal gland } & \multicolumn{2}{|c|}{ Pituitary gland } & \multirow{3}{*}{$\begin{array}{c}\text { Body } \\
\begin{array}{c}\text { Weight } \\
(\mathrm{g})\end{array} \\
210 \pm 10\end{array}$} \\
\hline & \multicolumn{2}{|c|}{ (nmol/glands) } & \multicolumn{2}{|c|}{ (nmol/glands) } & \\
\hline & $6.70 \pm 3.58$ & $3.81 \pm 0.78$ & $2.45 \pm 1.05$ & $13.98 \pm 3.76$ & \\
\hline Fasting for 2 days & $2.53 \pm 0.90^{*}$ & $5.70 \pm 1.71$ & $0.14 \pm 0.07 * *$ & $13.82 \pm 2.60$ & $168 \pm 6^{* *}$ \\
\hline Fasting for 3 days & $2.38 \pm 1.00^{*}$ & $1.79 \pm 0.76$ & $0.58 \pm 0.18^{* *}$ & $12.20 \pm 3.71$ & $127 \pm 5^{* *}$ \\
\hline
\end{tabular}

All values are expressed as mean $\pm \mathrm{SD}(\mathrm{N}=4) .^{*}: \mathrm{p}<0.05,{ }^{* *}: \mathrm{p}<0.01 \mathrm{VS}$. control group. 

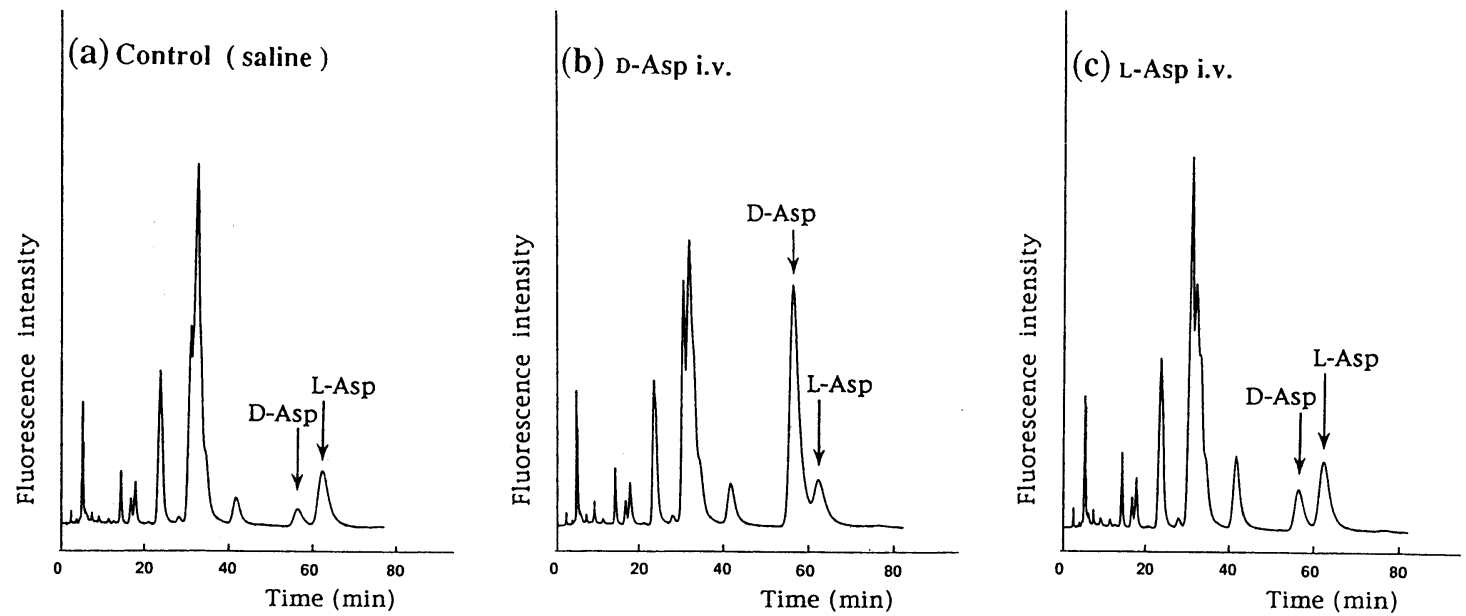

Fig. 1. Chromatograms of $\mathrm{MeOH}$ extracts obtained from pineal glands of rats at $30 \mathrm{~min}$ after administration of saline $(10 \mathrm{ml} / \mathrm{kg})$ (a), D-Asp (50 mg/kg) (b) and L-Asp (50 mg/kg) (c), respectively.
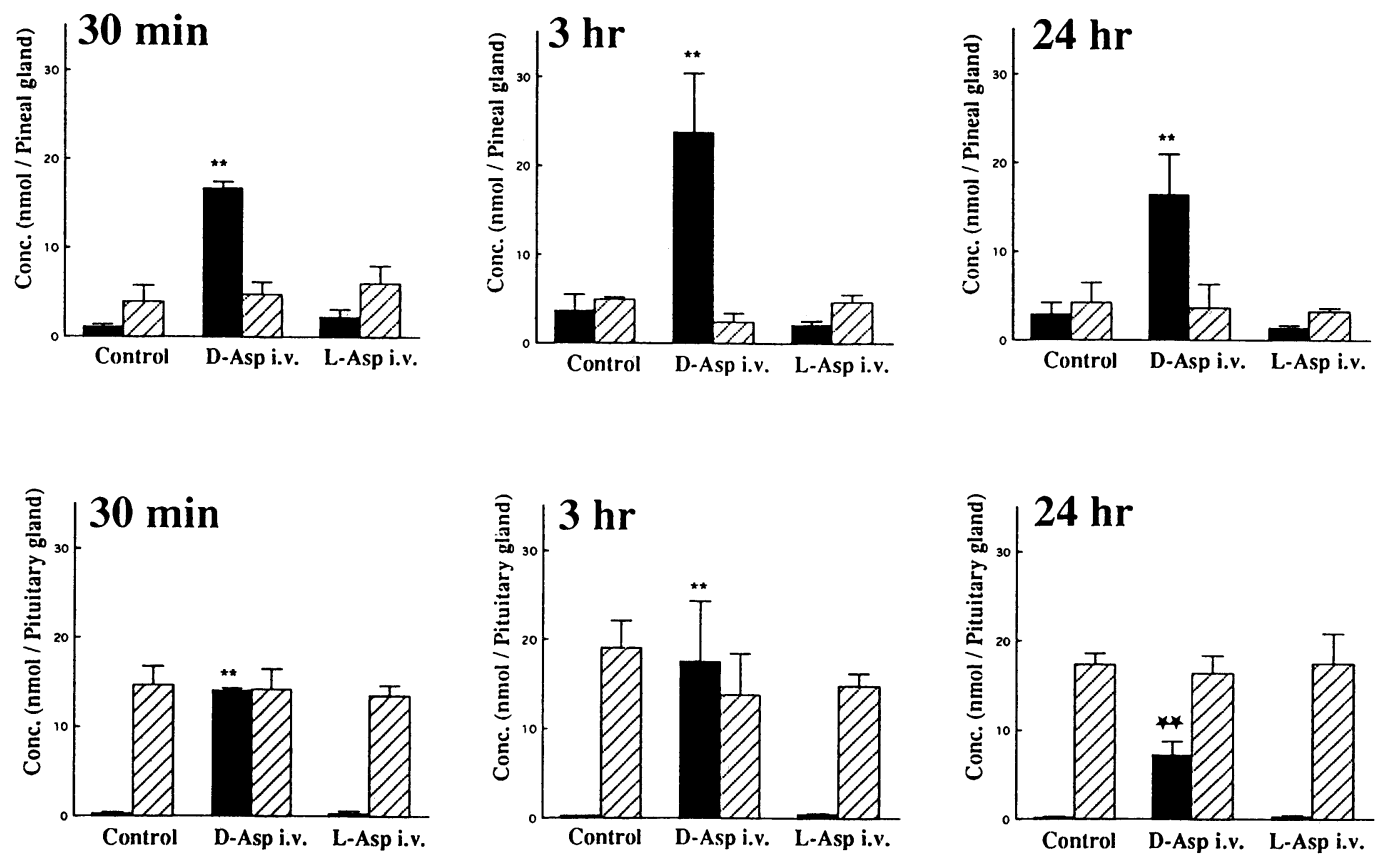

D-Asp Z L-Asp

Fig. 2. D- and L-Asp concentration in pineal (upper) and pituitary glands (lower) of rats at $30 \mathrm{~min}, 3 \mathrm{hr}$ and $24 \mathrm{hr}$ after intravenous administration' of saline $(10 \mathrm{ml} / \mathrm{kg}), \mathrm{D}-\mathrm{Asp}(50 \mathrm{mg} / \mathrm{kg})$ and L-Asp $(50 \mathrm{mg} / \mathrm{kg}) . \star \star ; \mathrm{p}<0.01$ versus control.

of D-Asp present in vivo in these tissues originated from an exogenous source, such as the daily food intake which contained D-Asp. In order to further explore the origin of D-Asp, in the present work, the effect of fasting was first investigated on the concentration of D-Asp present in vivo in pineal and pituitary glands.
The decrease of D-Asp concentration was clearly observed by fasting since the values $6.70 \pm 3.58$ decreased to $2.38 \pm 1.00 \mathrm{nmol} / \mathrm{pineal}$ gland $(\mathrm{p}<0.05)$, and $2.45 \pm 1.05$ to $0.58 \pm 0.18 \mathrm{nmol} / \mathrm{pituitary}$ gland $(\mathrm{p}<0.01$, Table I), supporting further the idea that D-Asp in the diet is one of the supply source of D-Asp in pineal and pituitary glands. 

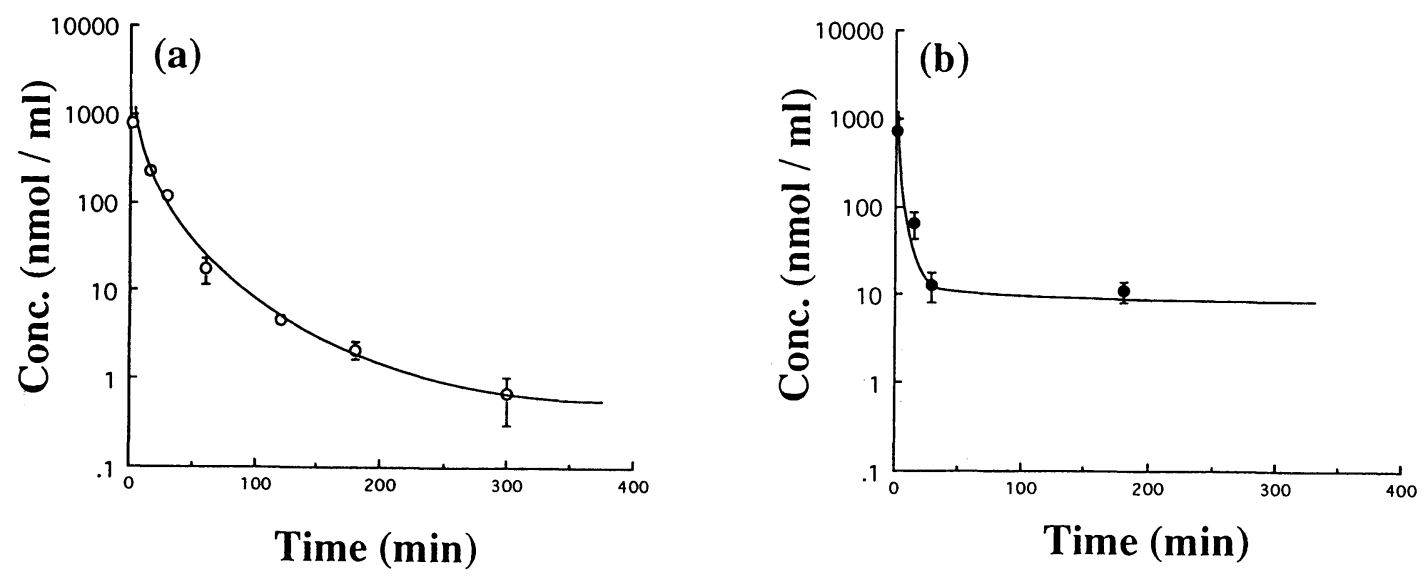

Fig. 3. D- and L-Asp concentration in plasma of rats after intravenous administration of D-Asp (50 mg/kg) (a) and L-Asp $(50 \mathrm{mg} / \mathrm{kg})(\mathrm{b})$.

However, the fact that the level did not reach to null even after 3 days fasting suggests that the possibility of biosynthesis in vivo could not be excluded. Although Dunlop and Neidle ${ }^{10)}$ reported recently that D-Ser was produced from L-Ser in the rat forebrain in vivo, to our knowledge, there is no report on the biosynthesis of D-Asp in mammals. Further studies have to be taken to clarify the issue of biosynthesis.

No remarkable change of L-Asp concentration observed under fasting may be attributed to the biosynthesis of L-Asp in vivo, or the release of L-Asp from a storage site in the other tissues such as liver. In the previous work, it was not clarified whether the long deposit of radioactivities in pineal and pituitary glands were derived from ${ }^{14} \mathrm{C}$-D-Asp itself or from the radioactive metabolite(s). ${ }^{14)}$ Thus, in this experiment, the fate of DAsp itself was examined by HPLC after intravenous administration of cold D-Asp. As was observed in the previous experiment, a large amount of D-Asp already appeared and accumulated in pineal and pituitary glands at $30 \mathrm{~min}$ after the administration. The chemical identification clearly demonstrated the long deposit of D-Asp in both the glands. The levels in these glands hardly changed from 30 $\min$ to $24 \mathrm{hr}$ (16,7-16.5 nmol/pineal glands, 14.0-7.22 $\mathrm{nmol} /$ pituitary gland), although the plasma concentration of D-Asp approached to the control level from $3 \mathrm{hr}$ after administration, indicating that D-Asp once incorporated from an extracellular fluid to both the glands was difficult to be metabolized and eliminated.

As for the incorporation of D-Asp from extracellular fluids to pineal and pituitary glands, it was suggested recently that it was mediated by the transporter for Lglutamate (Glu) in plasma membrane of pinealocyte cultured in the medium. ${ }^{16)}$ The in vivo incorporation of $\mathrm{D}$ -
Asp from extracellular fluids into both the glands might be attributed to the same transporter.

In the case of L-Asp administration, no accumulation in these glands were observed. This is mainly due to that L-Asp is metabolized instantaneously to the other substances in mammalian body. Moreover, the administration of L-Asp gave no detectable change of D-Asp concentration in pineal and pituitary glands, indicating that the possibility of in vivo epimerization of L-Asp to D-Asp in the glands could be excluded.

These results obtained in the present study indicate that D-Asp once incorporated from extracellular fluids to pineal and pituitary glands is difficult to be metabolized and eliminated, whereas L-Asp is immediately metabolized to the other compounds. The resistance to the leak of the incorporated D-Asp was also verified by in vitro experiment where only $50 \mu \mathrm{M}$ digitonin which permeabilize the plasma membrane allowed the release of D-Asp from pinealocyte to the outer medium. ${ }^{16)}$

Further studies are now underway on the metabolism of D-Asp in pineal and pituitary glands and the details will appear elsewhere.

Acknowledgements. The authors thank Dr. Chang-kee Lim for his kind advice on preparing the manuscript. Thanks are also due to Sumika Chemical Analysis Center, Co., Ltd. for the generous gift of Sumichiral OA-2500 (S) and (R).

\section{References}

1) Corrigan, J. J. (1969) Science 164, 142-149.

2) Imai, K., Fukushima, T., Santa, T., Homma, H., Hamase, K., Sakai, K., and Kato, M. (1996) Biomed. Chromatogr. 10, 303-312. 
3) Dunlop, D. S., Neidle, A., Mchale, D., Dunlop, D. M., and Laitha, A. (1986) Biochem. Biophys. Res. Commun. 141, 27-32.

4) D'Aniello, A., D’Onofrio, G., Pischetola, M., D’Aniello, G., Vetere, A., Petrucelli, L., and Fisher, G. H. (1993) J. Biol. Chem. 268, 26941-26949.

5) Hashimoto, A., Nishikawa, T., Oka, T., Hayashi, T., and Takahashi, K. (1993) FEBS lett. 331, 4-8.

6) D'Aniello, A., Cosmo, A. D., Cristo, C. D., Annunziato, L., Petrucelli, L., and Fisher, G. (1996) Life Sci. 59, 97-104.

7) Hashimoto, A., Kumashiro, S., Nishikawa, T., Oka, T., Takahashi, K., Mito, T., Takashima, S., Doi, N., Mizutani, Y., Yamazaki, T., Kaneko, T., and Ootomo, E. (1993) J. Neurochem. 61, 348-351.

8) Hashimoto, A., Oka, T., and Nishikawa, T. (1995) Eur. J. Neurosci. 7, 1657-1663.

9) Schell, M. J., Brady, R. O., Molliver, M. E., and Snyder, S. H. (1997) J. Neurochem. 17, 1604-1615.
10) Dunlop, D. S., and Neidle, A. (1997) Biochem. Biophys. Res. Commun. 235, 26-30.

11) Imai, K., Fukushima, T., Hagiwara, K., and Santa, T. (1995) Biomed. Chromatogr. 9, 106-109.

12) Hamase, K., Homma, H., Takigawa, Y., Fukushima, T., Santa, T., and Imai, K. (1997) Biochim. Biophys. Acta 1334, 214-222.

13) Schell, M. J., Cooper, O. B., and Snyder, S. H. (1997) Proc. Natl. Acad. Sci. U.S.A. 94, 2013-2018.

14) Imai, K., Fukushima, T., Santa, T., Homma, H., Sugihara, J., Kodama, H., and Yoshikawa, M. (1997) Proc. Japan Acad. 73B, 48-52.

15) Kera, Y., Aoyama, H., Matsumura, H., Hasegawa, A., Nagasaki, H., and Yamada, R. (1995) Biochim. Biophys. Acta 1243, 282-286.

16) Yatsushiro, S., Yamada, H., Kozaki, S., Kumon, H., Michibata, H, Yamamoto, A., and Moriyama, Y. (1997) J. Neurochem. 69, 340-347. 